\title{
The Harness Workbench: Unified and Adaptive Access to Diverse HPC Platforms
}

\author{
U.S. Department of Energy Grant DE-FG02-06ER25728 \\ Final Progress Report \\ George Bosilca \\ Department of Electrical Engineering and Computer Science \\ University of Tennessee, Knoxville, TN 37996, USA \\ bosilca@icl.utk.edu
}

\section{Overview}

The primary goal of the Harness workbench project $(H W B)$ was to investigate innovative software environments that help enhance the overall productivity of science applications on large and diverse HPC platforms. Two complementary frameworks were envisioned:

1. a virtualized command toolkit ( $V C T$ ) for application building, deployment, and execution, which provides a common view across diverse HPC systems, in particular the DOE leadership computing platforms (Cray, IBM, SGI, and clusters);

2. a unified runtime environment $(R T E)$ that consolidates access to runtime services via an adaptive framework for execution-time and post processing activities.

This project was a joint effort between Emory University, Oak Ridge National Laboratory (ORNL), and the University of Tennessee. The three institutions involved in this project worked closely together and the project methodology was driven by regular meetings of all personnel, where technical design, operational modes, compatibility aspects, and integration issues were discussed in detail. The sub-projects undertaken by each collaborator were closely linked and addressed complementary aspects of the global project. This report presents the work realized during the entire period of the proposed work at the University of Tennessee.

\section{Overall Progress}

The main effort undertaken at the University of Tennessee, Knoxville focused on the Run Time Environments (RTE) and the unified parallel debuggers interface. Most of our efforts targeted the Open RTE, augmenting its capabilities such as fault tolerance and scalability. Our efforts resulted in many published papers, a high level API for the interface between parallel applications and debuggers, multiple scalability and fault tolerance improvements in the Open MPI RTE, and a large number of external collaborations covering different aspects of the work realized in this context. 


\subsection{High Level API for Harness RTE}

The Run Time Environment (RTE) layer is essentially the interface between the user and application on the one hand, and the batch scheduler, native operating system and hardware platforms on the other. Even though it executes on the target machine, the RTE is considered part of the Harness workbench since it is operated, controlled, and configured from within the workbench. The RTE is responsible for actions such as process spawning and management, staging files, accessing schedulers, queuing jobs, monitoring execution, returning results, and reporting status. Some of these actions are initiated or controlled in the preparatory stage, but are carried out in conjunction with RTE support. The role of the RTE is to provide a uniform set of services that virtualize the capabilities of the underlying Operating Environments. These services were proposed as external interfaces to the users via the VCT substrate and also by other entities (e.g., PTP/Eclipse).

The Generalized Harness RTE would be responsible for interpreting service requests from the front-end systems, such as VCT or PTP/Eclipse, and mapping them to appropriate commands on the specific HPC platforms via either direct, native, platform specific invocations or through another RTE back-end plugin. Additionally, the capabilities offered to the front ends can also be extended dynamically by allowing them to load specialized modules into the Harness RTE. Moreover, we investigated the possible addition into this level of a generic service targeted to file manipulations. This service exposes a common interface on all operating systems (similar to the POSIX interface), which is completely independent of the underlying file system. Unlike MPI-IO, this service is not used during the parallel application execution, its usage is restricted at startup and shutdown to move files to and from the target platform. Such a service can have multiple usages, either staging the application into the execution nodes or moving the checkpoint to and from the reserved nodes.

\subsection{Scalability and Fault Management}

Support for fault management varies among RTE systems. Unfortunately, in today's HPC world, fault management is still a underused capability, and the runtime environments barely propose such features. The most basic ones do not provide any fault management at all, while more advanced RTEs are able to detect faults of system components and possibly trigger appropriate fault tolerance mechanisms. Expanding such capabilities allows the creation of sophisticated RTEs that not only detect failures, but are aware of their impact and can extend recovery and/or high availability mechanisms to applications and middle-ware. Such mechanisms may be completely transparent to the application, such as with the BL/CR checkpoint/restart layer, or may require additional fault handling by the application, as is the case with FT-MPI and Open MPI. In both cases, active/warm stand-by high availability is provided through automatic failure detection and adaptation, where some application state is lost by rolling back to an earlier state (checkpoint). Active/hot standby solutions, such as MPICH-V and Open MPI, and active/active solutions like the HARNESS distributed virtual machine, typically supply a highly available communication substrate using advanced commit protocols, such as message logging and distributed control, to allow automatic failure detection and adaptation without any loss of state.

The Harness Run Time Environment research had the goal of providing a virtualiza- 
tion layer for fault tolerance and high availability in HPC environments by providing hooks to existing solutions. For example, an application may be able to recover using a checkpoint/restart layer, but the automatic restart of the application might still require the HRTE to stage the checkpoint to the new location and resubmit the failed job. Furthermore, in order for the failure to be recognized by the higher-level recovery layer, appropriate detection and notification mechanisms are needed. Virtualization of these mechanisms via plugins, capable of implementing the necessary functionality if not directly supported by the underlying RTE, will facilitate development of portable fault-tolerance systems. This mechanism will also alleviate the need to adapt applications to a specific platform and to the particular failure-handling mechanisms being employed. Our virtualization effort has built upon recent research advances in reliability, availability and serviceability (RAS) for HPC, performed within our group as well as within the MOLAR project.

Common interfaces for some of the most used environments supporting fault management have been identified. We developed, improved, and maintained a fault aware layer in the existing run-time environment Open RTE. As a result, Open RTE now supports multiple types of recovery (i.e., transparent or not) and is able to integrate tightly with several batch scheduling systems. Handling fault is only a part of the tasks of an RTE, which is activated only in the event where a fault occurs. During normal execution, the mechanisms involved in the fault management and recovery are expected to stay out of the execution path in such a way that no performance loss will affect the parallel application. Additionally, we focused our efforts on improving existing transparent fault management approaches for parallel applications (mainly based on MPI). As a result, the cost of transparent fault management based on a pessimistic message logging approach has greatly improved, lowering the overall cost of fault tolerance to under $5 \%$ as proved by the paper published at ISC'08. Therefore, it is possible to claim that large scale fault tolerant applications are now a viable option for the HPC field.

Proposing a viable checkpoint/restart was a partial solution of the larger goal. Before proposing means to recover applications from faults, the same underlying techniques can be used to propose an interface for debugging large scale applications. In this context, we focused on two complementary approaches, both having the potential to improve the debugging experience significantly from the user perspective, and therefore improve the timeto-solution for scientific applications.

The first approach improves the integration between a parallel debugger and the MPI library used by parallel applications. The MPI library can export internal information allowing the debugger to provide pertinent information to the user, such information having the potential to help the developers understand coding issues.

The second approach focuses on the user's capability to roll back a parallel application to a state where the problem generating the analyzed error hasn't yet occurred. In other terms, when the user detects an error it is usually difficult to trace the execution steps (in terms of exchanged messages between the peers) that led to the erroneous state. Based on the strategies developed for checkpoint/restart, we were able to roll back the application to a previous state and allow the user to re-execute the application step-by-step, improving his or her ability to trace and eventually understand the root of the problem that generates the fault in the application.

A prototype implementation has been completed in the context of Open MPI and To- 
talView. For example, in the graphical interface of TotalView, a user can mouse-over any MPI defined object (such as MPI_Communicator and MPI_Request), and see the internal state. For a communicator, Open MPI is able to provide information about the number of nodes in the communicator, the rank of the current process in this communicator, as well as all the pending requests previously posted and not yet completed. For a request, the state of the request is available to the user, indicating how much data has been sent, as well as some MPI related information such as the request tag and peer. A paper is currently being prepared for submission that will describe this interface and how it can be implemented in both MPI libraries and parallel debuggers. Contacts have been made with several MPI library developers (Open MPI and MPICH), and with parallel debuggers developers (TotalView, Alinea, and Eclipse), such that the integration of such interfaces are now a reality. Moreover, the work realized in this context has been presented in front of the tools working group in the MPI forum, and some of the ideas were proposed for inclusion in the MPI 3.0.

\section{Research Output}

Our investigations of the Run Time Environments, the communication layer connecting all the resources involved in the successful execution of the parallel application, and the parallel debugging techniques have been published in several papers. Below is the exhaustive list of published papers resulting from the work done in the context of the Harness project.

- Performance Analysis of MPI Collective Operations, Jelena Pješivac-Grbović, Thara Angskun, George Bosilca, Graham E. Fagg, Edgar Gabriel, and Jack J. Dongarra, Cluster Computing Journal, Volume 10, pp 127-143, 2007.

- MPI Collective Algorithm Selection and Quadtree Encoding, Jelena Pješivac-Grbović, Graham E. Fagg, Thara Angskun, George Bosilca, Jack J. Dongarra, accepted in Parallel Computing, May 2007.

- Disaster Survival Guide in Petascale Computing: An Algorithmic Approach, Jack J. Dongarra, Zizhong Chen, George Bosilca, and Julien Langou, to appear in Petascale Computing: Algorithms and Applications, Chapman \& Hall / CRC Press.

- Binomial Graph: A Scalable and Fault-Tolerant Logical Network Topology, Thara Angskun, George Bosilca, Jack Dongarra, to be published in the Fifth International Symposium on Parallel and Distributed Processing and Applications (ISPA-07) to be held in Niagara Falls, Canada, August 29-31, 2007 and published in Springer's Lecture Note in Computer Science (LNCS) Accepted May 2007.

- Reliability Analysis of Self-Healing Network using Discrete-Event Simulation, Thara Angskun, George Bosilca, Graham Fagg, Jelena Pješivac-Grbović, and Jack J. Dongarra, CCGrid07.

- Decision Trees and MPI Collective Algorithm Selection Problem, Jelena PješivacGrbović, Graham E. Fagg, Thara Angskun, George Bosilca, and Jack J. Dongarra, To appear in EuroPar 2007. 
- Self-Healing in Binomial Graph Networks, Thara Angskun, George Bosilca and Jack Dongarra, 2nd International Workshop On Reliability in Decentralized Distributed Systems (RDDS 2007), Vilamoura, Algarve, Portugal, Nov, 2007.

- Optimal Routing in Binomial Graph Networks, Thara Angskun, George Bosilca, Brad Vander Zanden and Jack Dongarra, The International Conference on Parallel and Distributed Computing, Applications and Technologies (PDCAT), Adelaide, Australia, Dec, 2007.

- Retrospect: Deterministic Replay of MPI Applications for Interactive Distributed Debugging, Aurelien Bouteiller, George Bosilca and Jack Dongarra, Euro PVM/MPI, 2007.

- Redesigning the Message Logging Model for High Performance, Aurelien Bouteiller, George Bosilca and Jack Dongarra, accepted in ISC'08.

- Constructing Resilient Communication Infrastructure for Runtime Environments, George Bosilca,Camille Coti,Thomas Herault, Pierre Lemarinier, Jack Dongarra, Technical Report ICL-UT-09-02

- Self-healing network for scalable fault-tolerant runtime environments, Thara Angskun and Graham Fagg and George Bosilca and Jelena Pjeivac-Grbovi and Jack Dongarra, FGCS 2010

- On Scalability for MPI Runtime Systems, Bosilca, G. and Herault, T. and Rezmerita, A. and Dongarra, J., IEEE International Conference on Cluster Computing (CLUSTER), 2011

\section{Summary}

In this project, we conducted preliminary research to create a flexible environment that encapsulates the knowledge of the application developers, site system administrators, and the vendors to assist application building and execution on HPC systems, in particular the DOE leadership computing platforms. The key research involved how to describe and use the knowledge from these varied sources to improve productivity of the end-user scientists, while creating a flexible and modular environment supporting all these features. 